\title{
Flow Blurring-Enabled Production of Polymer Filaments from Poly(ethylene oxide) Solutions
}

\author{
Luis B. Modesto-López,*둘 Agustín Pérez-Arjona, and Alfonso M. Gañán-Calvo ${ }^{\circ}$ \\ Department of Aerospace Engineering and Fluid Mechanics, University of Seville, Camino de los Descubrimientos S/N, 41092 \\ Seville, Spain
}

\section{Supporting Information}

\begin{abstract}
Flow blurring (FB) atomizers are relatively simple yet robust devices used for the generation of sprays from solutions of a wide range of viscosities. In this work, we have demonstrated that FB devices may also be applied for massive production of liquid filaments from polymeric solutions. They can later be transformed into solid filaments and fibers, leading to the production of so-called fiber mats. The liquid precursors consisted of poly(ethylene oxide) (PEO) solutions of varying molecular weights $\left(10^{5}\right.$ [100k to $\left.4 \times 10^{6} \mathrm{~g} / \mathrm{mol}[4 \mathrm{M}]\right)$ and concentrations. The FB device was operated in the gas pressure range of 3-6 bar. Except for solutions of PEO 100k, all solutions exhibited a shear thinning behavior. For massive filament production, a threshold

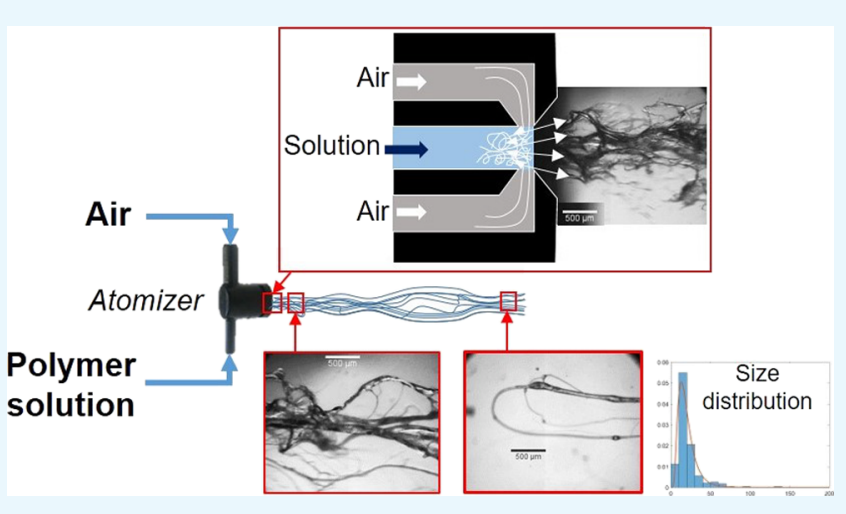
polymer concentration $\left(c_{\mathrm{t}}\right)$ was identified for each molecular weight. Below such concentration, the atomization resulted in droplets (the classical FB functioning mode). Such a threshold value decreased as the PEO molecular weight increased, and it coincides with the polymer coil overlap concentration, $c^{*}$. The viscoelastic nature of the solutions was also observed to increase with the molecular weight. A 3.2 dependency of the zero-shear rate viscosity on a so-called Bueche parameter was found for filament production, whereas a nearly linear dependency was found for droplet production. In general, the mean diameter of the filaments decreased as they traveled downstream from the atomization point. Furthermore, at a given distance from the atomizer outlet and gas pressure, the mean filament diameter slightly shifted toward larger sizes with increasing PEO molecular weight. The tendency agrees well with the calculated filaments' Deborah number, which increases with PEO molecular weight. The approach presented herein describes a highthroughput and efficient method for the massive production of viscous filaments. These may be transformed into fibers by an on-line drying step.
\end{abstract}

\section{INTRODUCTION}

Liquid filaments from polymeric solutions or melts constitute the base for fabrication of microfiber networks, so-called fiber mats, in various processes. These mats, and their derivatives, find a wide range of applications as high-value fabrics, from scaffolds for biomaterials and tissue engineering, ${ }^{1,2}$ to films for energy harvesting ${ }^{3}$ and electronic devices. ${ }^{4}$ They also serve as catalytic surfaces in the chemical industry. ${ }^{5,6}$ The generation method of the filaments affects the final characteristics of the microstructured fiber mats, such as the diameter of the fibers and their composition. In general, the liquid filaments are transformed into fibers by solidification because of cooling, in the case of polymer melts, or by solvent evaporation in the case of polymer solutions. Processing of polymers is of interest to many areas of material science, for instance in high-tech applications such as space technology. ${ }^{7,8}$

Common approaches for generation of micrometer-sized liquid filaments or jets involve electrical forces, as in electrospinning. ${ }^{5,9,10}$ In such techniques, a meniscus of a viscous liquid, continuously emanating from a capillary tube, is transformed into a so-called Taylor cone by the action of a sufficiently high electric potential. At this critical potential, the cone's apex emits a jet that travels toward an electrically grounded electrode positioned downstream of the capillary. More details of the electrospinning and electrically driven liquid jets can be found elsewhere. ${ }^{5,11-17}$ Other methods use aerodynamic forces to focus a liquid stream through an orifice, thus forming a relatively thin, uniform jet in what is known as flow focusing (FF). ${ }^{18-20}$ In general, a three-dimensional FF device consists of two concentric capillary tubes, where the liquid flows in the inner capillary and a gas in the exterior one. At the capillaries' end, the gas surrounds the liquid stream and focuses it to pass through a small orifice, perforated on a plate and placed downstream of the tubes' end, thus generating a jet. These methods are advantageous for production of liquid jets

Received: September 27, 2018

Accepted: January 14, 2019

Published: February 5, 2019 

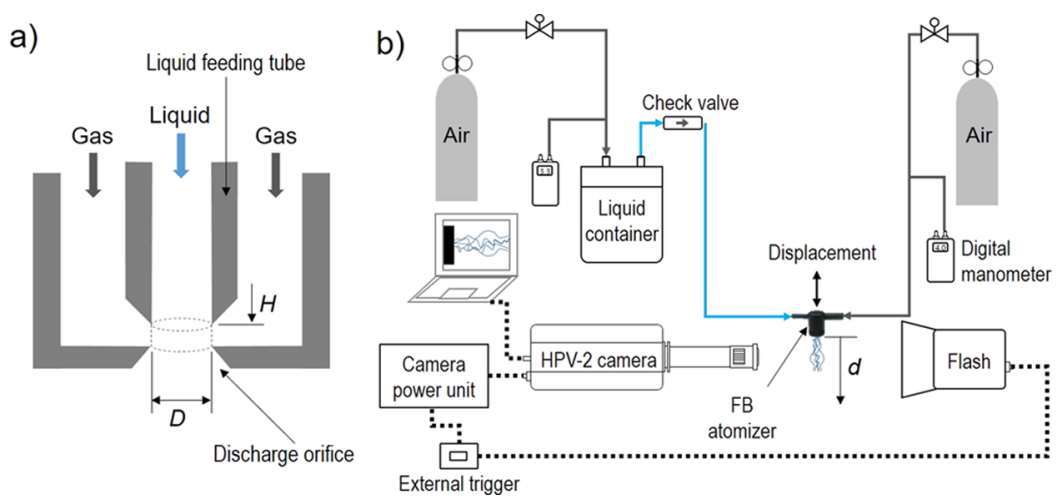

Figure 1. (a) Schematic of the internal configuration of a typical FB atomizer; (b) experimental setup for atomization of PEO aqueous solutions.

with uniform diameter at the expense of a relatively low throughput.

However, in this work, we demonstrate that the so-called flow blurring (FB) method, ${ }^{18}$ a pneumatic approach for atomization of liquids into fine sprays, may also be used for the massive production of liquid filaments. The FB technique has a throughput of the order of tens of liters per hour, thus allowing mass production of filaments at a rate many orders of magnitude larger than most common methods. The mechanism of liquid atomization by FB was first reported by GañanCalvo ${ }^{18}$ and since then the technique has been employed by others. ${ }^{19,21-24}$ Schematics of a typical FB atomizer are shown in Figure 1a. Geometrically, an FB atomizer is similar to an FF device, the only difference being the value of a dimensionless parameter $\phi=H / D$, where $D$ is the diameter of the discharge orifice and $H$ is the gap between the tip of the liquid feeding tube and the discharge orifice (see Figure 1a). For $\phi$ approximately $>0.25$, the FF mode is achieved, whereas for $\phi$ below such value the device operates in FB mode. ${ }^{18}$ Briefly, in an FB device, the kinetic energy carried by a gas flow radially imploding onto the liquid stream induces a relatively stagnant region between the feeding tube and the outlet, thus generating a turbulent back-flow pattern in the interior of the device, which promotes an efficient gas-liquid interaction and simultaneously results in the breakup of the liquid surface to form droplets. This characteristic flow constitutes one of the main advantages of the FB method as it is responsible for its high atomization efficiency. Indeed, FB devices generate from five to fifty times more surface area than other methods, such as plain-jet air-blast and effervescent atomizers. ${ }^{18,22,25} \mathrm{FB}$ is a relatively simple yet robust method to atomize a variety of liquids with a wide range of viscosities, from water and ethanol $\left(\sim 10^{-3} \mathrm{~Pa} \cdot \mathrm{s}\right),{ }^{21}$ to vegetable oil $(\sim 500 \mathrm{~Pa} \cdot \mathrm{s}) .^{22}$ So far, FB has only been employed to disperse bulk liquids into droplets, and not to produce liquid filaments from polymer solutions. These are known to exhibit viscoelastic nature depending on their molecular weight and concentration.

Viscoelastic liquids are fluids that exhibit an extensional rheology, thus varying their viscosity with time and applied strain rate. $^{26-28}$ The length of the chain, that is, the molecular weight of the polymer, is the main factor determining its rheological properties. ${ }^{28}$ It is this elastic characteristic of polymeric solutions which leads us to think that, under the relative high stress experienced by the liquid stream in the FB atomizer, it may exhibit an elongational flow behavior rather than breaking up into droplets. The work of Keshavarz and coworkers also shows the formation of short-range ligaments during the fragmentation of dilute viscoelastic liquids. ${ }^{29}$ Some authors have reported the formation of filaments or fibers from highly viscous liquids using air-blast type of atomizers under certain conditions of applied pressure and temperature. Lysak and collaborators reported the production of fibers, using a pneumatic atomization method, from polymer melts, which requires additional energy to heat the polymer and achieve its flowing state. ${ }^{30}$ Nevertheless, details of their atomizer and its functioning mechanism remain largely unknown, but it can be inferred that they used a type of air-blast atomizer. Otaigbe and McAvoy atomized a molten stream of polyethylene using an air-blast device combining high-pressure ( $>72$ bar or $7.6 \mathrm{MPa}$ ) and temperature $\left(\sim 200{ }^{\circ} \mathrm{C}\right)$, and reported the formation of fibers under certain conditions. ${ }^{31} \mathrm{Li}$ observed the formation of ligaments and filamentary structures during air-blast atomization of relatively low molecular weight poly(ethylene oxide) (PEO) and pointed out that the physical scale of these ligaments increased with PEO molecular weight and thus elasticity. ${ }^{32}$ Solution blow spinning (SBS) has also been used to produce polymer fibers without the need of an electric field. ${ }^{33}$ Daristotle and co-workers ${ }^{33}$ summarized the important parameters that govern the SBS process and have identified a critical polymer concentration for fiber formation, which is also shared with the electrospinning process.

In the present work, we have used aqueous solutions of PEO of various molecular weights and concentrations to demonstrate the massive formation of filaments at a critical concentration using the FB method, at room temperature, even at distances far downstream of the atomization point.

\section{EXPERIMENTAL SECTION}

2.1. Materials. $\mathrm{PEO}$ of viscosity-average molecular weight, $M_{\mathrm{v}}, 100000 \mathrm{~g} / \mathrm{mol}$ (PEO 100k), $600000 \mathrm{~g} / \mathrm{mol}$ (PEO 600k), $1000000 \mathrm{~g} / \mathrm{mol}$ (PEO 1M), $2000000 \mathrm{~g} / \mathrm{mol}$ (PEO 2M), and $4000000 \mathrm{~g} / \mathrm{mol}$ (PEO 4M) were purchased from SigmaAldrich and used as received. Distilled water (Milli-Q) was used as solvent. For atomization experiments, a commercial FB atomizer (Ingeniatrics Tecnologias, Seville, Spain) with an orifice diameter $(D)$ of $700 \mu \mathrm{m}$ and a distance from the liquid capillary outlet to the orifice $(H)$ of $100 \mu$ m was used, resulting in a $\phi$ of $1 / 7 .^{19,21}$

2.2. Solution Preparation, Viscosity, and Surface Tension Measurements. Polymeric solutions of different concentrations were prepared by mixing the appropriate amount of PEO in distilled water, followed by mixing with a magnetic stirrer until a uniform solution was obtained. Generally, the final aqueous polymeric solutions were trans- 
Table 1. Properties of PEO Solutions at Threshold Concentration, $c_{t}$

\begin{tabular}{|c|c|c|c|c|c|c|c|c|c|}
\hline$M_{\mathrm{v}}(\mathrm{g} / \mathrm{mol})$ & $c_{\mathrm{t}}($ wt $\%)$ & $\eta_{0}(\mathrm{~Pa} \cdot \mathrm{s})$ & $\sigma(\mathrm{N} / \mathrm{m})$ & $c_{\mathrm{m}} M_{\mathrm{v}} / M_{\mathrm{e}}(-)^{a}$ & $c^{*}(\mathrm{wt} \%)$ & $c_{\mathrm{e}}($ wt $\%)$ & $d_{\mathrm{j}}(\mu \mathrm{m})^{b}$ & $\lambda_{\mathrm{z}}(\mathrm{ms})$ & $\mathrm{De}(-)$ \\
\hline $100 \mathrm{k}$ & 7.0 & 0.0440 & 0.0652 & 1.59 & 3.00 & 17.11 & 16.3 & 0.0015 & 0.0018 \\
\hline $600 \mathrm{k}$ & 0.8 & 0.0108 & 0.0592 & 1.09 & 0.79 & 4.08 & 23.5 & 0.0360 & 0.0431 \\
\hline $1 \mathrm{M}$ & 0.5 & 0.0144 & 0.0587 & 1.14 & 0.54 & 2.71 & 33.5 & 0.0907 & 0.1070 \\
\hline $2 \mathrm{M}$ & 0.4 & 0.0111 & 0.0651 & 1.82 & 0.32 & 1.56 & 31.9 & 0.3110 & 0.3860 \\
\hline $4 \mathrm{M}$ & 0.2 & 0.0064 & 0.0620 & 1.82 & 0.19 & 0.89 & 32.7 & 1.070 & 1.290 \\
\hline
\end{tabular}

${ }^{a} M_{\mathrm{e}}=4400 \mathrm{~g} / \mathrm{mol} .{ }^{46}{ }^{b} d_{\mathrm{j}}$ is the mean filament diameter measured at $4 \mathrm{~cm}$ from the atomizer outlet and at gas pressure of 4 bar.

parent except for PEO 100k, for which a turbid liquid was obtained, probably due to its relatively high concentration. Typical stirring times were of the order of 1 day; in some cases, mild heating was applied to facilitate mixing, particularly in the case of PEO of higher molecular weight and relative high concentration. The solutions were cooled at room temperature prior to use. Subsequently, the viscosity was measured using a Brookfield DV-E instrument equipped with LV-type spindles, in the shear rate range of $1-100 \mathrm{~s}^{-1}$. All measurements were performed at room temperature $\left(19-24{ }^{\circ} \mathrm{C}\right.$ ) and ambient relative humidity $(40-60 \%)$. Solutions were allowed to stabilize for a few minutes before recording any measurement.

The surface tension of polymeric solutions in air was measured with a KSV contact angle meter (CAM 100) set up in a pendant drop configuration, for static measurements in the range $0^{\circ}-180^{\circ}$. The instrument is equipped with a FireWire video camera module with a resolution of $640 \times 480$ pixels and with a light-emitting diode, monochromatic, light source. The objective lens provided with the camera is telecentric with a 55 $\mathrm{mm}$ focus length. The instrument's software applies a curve fitting using the Young-Laplace equation to calculate surface tension. Only polymeric solutions with a critical concentration were measured (as described below).

2.3. FB Atomization and High-Speed Video Recording. Figure $1 \mathrm{~b}$ shows the setup used for atomization experiments. The FB atomizer was mounted on an optical table using high-precision, movable mounts (not shown in the figure), which allowed to displace it forward and backward along a straight center line. The atomizer was operated by controlling the pressures of gas, air in this case, and liquid supply lines. The air was fed into the FB atomizer directly from the supply line. The liquid was fed pneumatically thru a hermetic aluminum liquid container as depicted in the figure. A check valve was placed between the liquid container and the atomizer inlet to avoid back flow. Both, gas and liquid pressures were measured by digital manometers. For the FB atomizer operation, first, the air pressure $\left(P_{\mathrm{g}}\right)$ was fixed in the range 3-6 bar, and then the liquid pressure $\left(P_{1}\right)$ was adjusted until a continuous, stable atomization was achieved. Typically, $P_{1}$ was $\sim 0.7$ bar lower than $P_{\mathrm{g}}$.

Videos of the atomization process were recorded at varying distances, $d$, from the emission point (i.e., the atomizer outlet, see Figure 1b) using a Shimadzu ultra-high-speed HPV-2 video camera capable of recording up to $10^{6} \mathrm{fps}$. The videos were illuminated with a high-intensity Walimex Pro Studio Flash (VC-4000) positioned on the opposite side of the camera, across the FB atomizer (Figure 1b). The flash was synchronized with the video camera's power unit through an external trigger. The camera began recording $1 \mathrm{~ms}$ after the trigger was manually switched on. ${ }^{15,21}$ The camera recording plane was focused along the centerline of the atomization output. The videos were then processed with the freeware image analysis software Image ${ }^{34}$ to obtain the diameter of filaments. To obtain the size distribution of filaments, several images recorded at the same $d$ and varying times were processed with ImageJ and a minimum of 150 filaments were measured. Only individual filaments were counted, that is, bundles whose filament diameters are indistinguishable were screened out.

\section{RESULTS AND DISCUSSION}

3.1. Viscoelastic Nature of PEO Aqueous Solutions. As the rheology of the solution plays an important role in liquid atomization processes, viscosity measurements were performed to investigate the solution characteristics at threshold concentrations ( $c_{\mathfrak{t}}$, summarized in Table 1$)$ at which liquid filaments are produced. These concentrations were identified in preliminary atomization experiments for each PEO molecular weight. The filaments remained present even at 4 $\mathrm{cm}$ downstream from the atomizer's outlet. We also show that these filamentary structures maintain such shapes even at distances as far as $8 \mathrm{~cm}$ from the emission point. For solution concentrations below $c_{\mathrm{t}}$, only droplets or a mixture of filaments and droplets are generated. Viscosity data of PEO solutions at this threshold concentration are depicted in Figure 2 as a

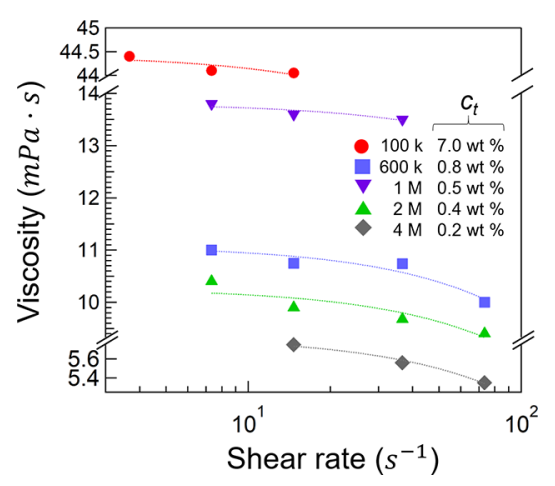

Figure 2. Viscosity of aqueous solutions of PEO, of varying molecular weight and concentration, as a function of shear rate.

function of shear rate. The figure indicates that PEO 100k $7 \mathrm{wt}$ $\%$ exhibits a Newtonian-like behavior within the measured shear rate range. Conversely, solutions of PEO of higher molecular weight show increased viscoelastic properties. Our results agree very well with more detailed measurements performed by other authors using PEO of similar molecular weights. $^{35}$ All solutions of PEO of $M_{\mathrm{v}}>100 \mathrm{k}$, in the concentration range used in this work, exhibit a shear thinning behavior. Ebagninin and co-workers ${ }^{35}$ and Grigorescu and Kulicke $^{36}$ identified a shear-thickening behavior of PEO aqueous solutions of a given critical concentration at relatively low shear rates. Such concentration increased with decreasing the molecular weight of PEO. ${ }^{35,36}$ However, the solutions used herein show a shear thinning behavior solely because their 
concentrations are below the critical value pointed out by Grigorescu and Kulicke ${ }^{36}$ and by Ebagninin et al. ${ }^{35}$ Note also that the shear thinning properties of the solutions used in this work are not as pronounced as it has been reported for aqueous solutions with a higher PEO concentration. ${ }^{35}$ It thus appears as though the viscosity of the solutions atomized with concentration $c_{\mathrm{t}}$, and which formed filaments, may approach its value at the so-called infinite-shear rate as a consequence of the peculiar stress concentration characteristic of the FB flow pattern.

Typically, polymer solutions exhibit five distinct states based on their mass composition in a thermodynamically good solvent. $^{35-39}$ To assess the rheological nature of our solutions, we have calculated the so-called overlap concentration, $c^{*}$, that is, the critical concentration above which the polymer coils overlap in solution (see, for instance, refs $^{38,41}$ for a detailed discussion on this topic). $c^{*}$ was estimated with $M_{\mathrm{v}} / R_{\mathrm{g}}{ }^{3} N_{\mathrm{A}}$ where $R_{\mathrm{g}}=0.0215 M_{\mathrm{v}}^{0.583}$ (as reported by Devanand and Selser ${ }^{42}$ ) is the radius of gyration (in $\mathrm{nm}$ ) of the polymer coil and $N_{\mathrm{A}}$ is Avogadro's number. ${ }^{40-43}$ In all calculations hereafter, $M_{\mathrm{v}}$ is given in units of $\mathrm{g} / \mathrm{mol}$. The values of the overlap concentration are summarized in Table 1 . The solution concentrations $(c)$ that we have identified as threshold values $\left(c_{\mathrm{t}}\right)$ to generate filaments with FB are of the order of $c^{*}, c_{\mathrm{t}} / c^{*}$ $\approx o(1)$, thus indicating that the polymer coil's overlap plays a key role in filament formation with FB. Similar results were obtained for other jetting processes such as electrospinning, ${ }^{44}$ although the physics behind the filament production process differs radically from the one presented herein. As in this work the ratio of atomized solution concentration to overlap concentration $c / c^{*} \approx 1$, they fall under the so-called semidilute regime. $^{36-39,45}$ In these types of semidilute solutions, intermolecular interactions and entanglements may become relevant. The nature of entanglements at concentrations above the so-called entanglement concentration, $c_{\mathrm{e}}$, leads to viscoelastic properties observed in shear flow experiments. ${ }^{36,37}$ Some authors have modeled the transition from dilute to concentrated solutions using a Bueche plot, that is, a graph of the zero-shear rate viscosity $\left(\eta_{0}\right)$ as a function of the product of concentration times the molecular weight of the polymer $(M)$. Generally, below a critical molecular weight, $\eta_{0}$ is directly proportional to $M$, and above such threshold, it is widely accepted that $\eta_{0}$ is proportional to $M^{3.4}$ for polymer melts. This viscosity increase has been attributed to intermolecular entanglement in the polymer chains. ${ }^{36}$

Figure 3 depicts the viscosity at zero-shear rate $\left(\eta_{0}\right)$ as a function of a dimensionless Bueche parameter $c_{\mathrm{m}} \cdot M_{\mathrm{v}} / M_{\mathrm{e}}$ for $\mathrm{PEO}$ in the range $100 \mathrm{k}$ to $4 \mathrm{M}$ measured in this work. In the figure, $c_{\mathrm{m}}$ is the polymer concentration given in mass fraction, $M_{\mathrm{v}}$ is its viscosity-average molecular weight, and $M_{\mathrm{e}}(4400 \mathrm{~g} /$ $\mathrm{mol})$ is the entanglement molecular weight of PEO in water. ${ }^{46}$ In the figure, the white squares represent data points of polymeric solutions (with $c_{t}$ ) which when atomized formed filaments (the ones indicated in Table 1). Two distinct regimes are distinguished, in agreement with literature reports. ${ }^{28,36,37} \mathrm{In}$ the first regime, $\eta_{0}$ is nearly directly proportional to the parameter $c_{\mathrm{m}} \cdot M_{\mathrm{v}} / M_{\mathrm{e}}$ up to a value of $\sim 1$, above which the viscosity increases sharply. For $c_{\mathrm{m}} M_{\mathrm{v}} / M_{\mathrm{e}}>1$, approximately a 3.2-dependency of $\eta_{0}$ on the dimensionless Bueche parameter is observed, which is very close to the value of 3.4 reported by others for the case of polymer melts (see Grigorescu and Kulicke $^{36}$ and Shenoy et al. $\left.{ }^{47}\right)$. In our work, the critical value of the Bueche parameter $\left(c_{\mathrm{m}} \cdot M_{\mathrm{v}} / M_{\mathrm{e}}\right)$ that results in atomization

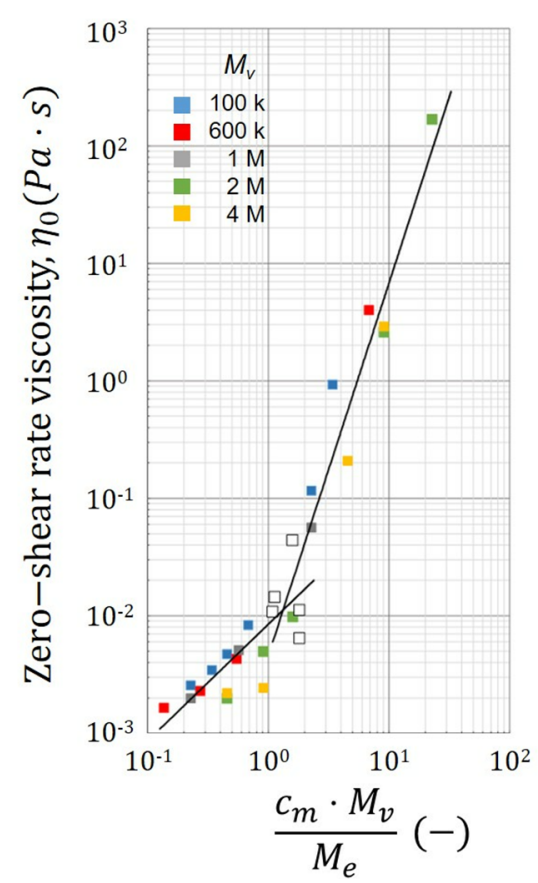

Figure 3. Zero-shear rate viscosity of PEO solutions as a function of the dimensionless parameter $c_{\mathrm{m}} M_{\mathrm{v}} / M_{\mathrm{e}}$. The white squares indicate data of polymers atomized into filaments (Table 1 ).

in the form of liquid filaments appears to be in the range $1-2$, thus resulting in $\eta_{0}$ of the order of $0.012 \mathrm{~Pa} \cdot \mathrm{s}$. As mentioned earlier, solutions with $c / c^{*}<1$, which correspond to $c_{\mathrm{m}} \cdot M_{\mathrm{v}} / M_{\mathrm{e}}$ $<1$ in Figure 2, are called dilute solutions and are not able to generate stable filaments with FB, implying that the filaments break up into smaller structures at short distances from the atomizer's outlet. It is thus clear that, under the FB conditions used in this work, only semidilute solutions produce stable filaments (with 3.2-dependency on $c_{\mathrm{m}} \cdot M_{\mathrm{v}} / M_{\mathrm{e}}$ ), which retain their structure even at distances as long as $8 \mathrm{~cm}(\sim 114 D)$ from the atomizer's outlet. We have calculated the so-called entanglement concentration, $c_{\mathrm{e}}$, the critical concentration above which the polymer coils begin to form entanglements, with $n_{\mathrm{e}}^{3(\nu-1)} /\left(M_{\mathrm{v}} A_{2}\right)$ for solutions that were atomized into filaments (see Table 1). ${ }^{42,45,48,49} n_{\mathrm{e}}$ is the number of monomers between entanglements and is calculated as the ratio of the molecular weight of entanglement to the molecular weight of the monomer, $M_{\mathrm{e}} / M_{\mathrm{o}}$. For PEO in water, the exponent $\nu=0.583$ and $A_{2}=0.0184 M_{\mathrm{v}}{ }^{-0.2}$ (in $\mathrm{mL} \mathrm{mol} / \mathrm{g}^{2}$ ). ${ }^{42}$ The condition that establishes the onset for polymer entanglement in solution is given by $c>c_{\mathrm{e}}$. As our data indicate, and as in our atomization experiments $c_{\mathrm{t}}(=c) / c^{*} \approx 1$, it follows that $c_{\mathrm{t}} / c^{*}<c_{\mathrm{e}} / c^{*}$; thus, the atomized solutions are semidiluted, unentangled solutions. Although the studied solutions do not form entanglements, the dependence of $\eta_{0}$ on $M_{\mathrm{v}}$ is thought to originate from coil overlapping.

3.2. Filament Production by FB-Based Atomization of PEO Aqueous Solutions. The PEO solutions were then systematically atomized with the FB device shown in Figure 1 at different gas pressures. It was observed that at each $P_{\mathrm{g}}$ a minimum value of liquid pressure is required to achieve a continuous and stable atomization of the solutions into liquid filaments. Such stability has a relatively strong dependence on $P_{1}$ as shown in Figure 4. The figure depicts images recorded right at the outlet of the $\mathrm{FB}$ device, $d=0 \mathrm{~cm}$, at the same gas pressure $\left(P_{\mathrm{g}}=4\right.$ bar $)$ but varying liquid pressures: (a) 3.3 and 

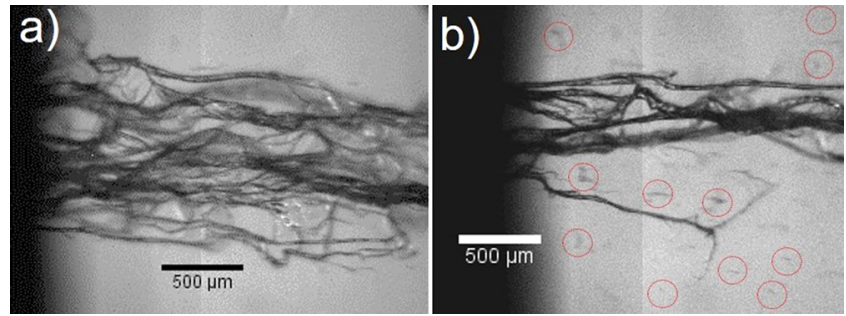

Figure 4. FB atomization of PEO 600k 0.8 wt $\%$ at $P_{\mathrm{g}}=4$ bar, (a) $P_{1}=$ $3.3 \mathrm{bar}$ and (b) $P_{1}=3.0$ bar.

(b) 3 bar. In Figure 4a, the liquid surface appears to be formed by filamentary structures solely, whereas in Figure $4 \mathrm{~b}$ smaller structures are also observed (red circles). These smaller structures, which most likely are generated in the atomizer's interior, transform into droplets downstream. Note also that their relative velocity appears to be higher than that of the filamentous structures, as observed in high-speed videos (not shown). Macroscopically, this "droplet" production regime exhibited a "cloud" surrounding a core filament bundle (and it was accompanied by a characteristic noise). However, when the liquid pressure was increased by 0.3 bar (Figure $4 a$ ), the cloud disappeared and only the filamentary structures were observed (while the noise also ceased). It is important to note that the formation of filaments at $d=0 \mathrm{~cm}$ is indicative that the FB is occurring, as expected, in the atomizer's interior, because they can only be the result of the breakup of the liquid surface.

Subsequently, PEO aqueous solutions were atomized at $P_{\mathrm{g}}=$ $3,4,5$, and 6 bar, with $P_{1}$ being the minimum to produce filaments (generally, $P_{1}$ was $\sim 0.7$ bar lower than $P_{\mathrm{g}}$ ). Figure 5 shows images of a typical atomization of an aqueous solution of PEO 600k $0.8 \mathrm{wt} \%$ recorded at varying distances $(d)$ from the atomizer outlet with a high-speed video camera. Right at the outlet $(d=0 \mathrm{~cm}$, Figure $5 \mathrm{a})$, the liquid surface is composed of filamentary structures which appear to be interconnected, and at this position it is difficult to discern the diameters of individual filaments. However, $0.5 \mathrm{~cm}$ downstream of the outlet some of those threads have separated from the core bundle, and there, several filament diameters may be measured. Nevertheless, in the atomization core, a few $(\sim 6)$ main "thick" bundles are observed, and smaller filaments also appear. Further downstream, the number of the core bundles is reduced as it is their typical diameter as can be observed in the images of Figure $5 \mathrm{c}-\mathrm{f}$. It is important to mention that the filamentary structures appear even at $d=8 \mathrm{~cm}$ from the emission point, which has not been reported before. In other words, the filaments do not break into droplets at $d=8 \mathrm{~cm}$. For instance, Li reported formation of filaments at distances as far as $4 \mathrm{~cm}$ from the atomizer outlet. ${ }^{32}$ The lifetime of filaments from polymeric solutions is associated to their extensional viscosity. It is known that addition of a high molecular weight macromolecule to a solvent such as water dramatically increases its extensional viscosity. The variation of the mean filament diameter as a function of $d$ can be quantitatively observed in the size distributions of Figure 6.

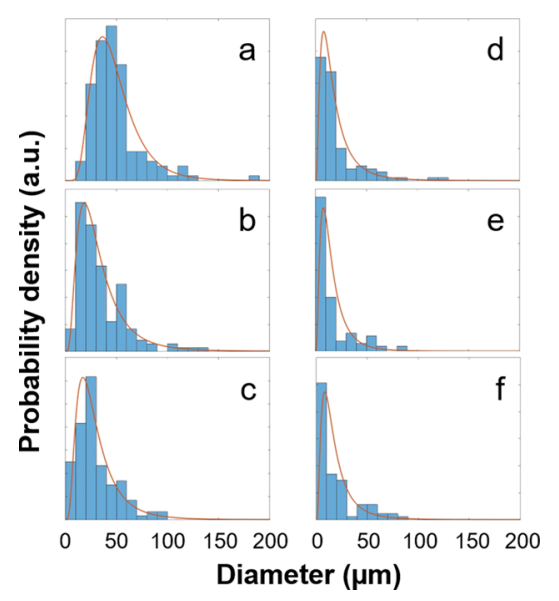

Figure 6. Size distribution of filaments from PEO 600k 0.8 wt \% atomized with an FB device at $P_{\mathrm{g}}=4$ bar and $P_{1}=3.3$ bar. $d=(\mathrm{a}) 0.5$, (b) 1 , (c) 2 , (d) 3 , (e) 4 , and (f) $8 \mathrm{~cm}$.

The trend indicates that the mean filament diameter shifts toward smaller sizes as the filaments travel downstream. At $d=$ $0.5 \mathrm{~cm}$, filaments' diameters are in the range $10-130 \mu \mathrm{m}$ and some of them have a size of $\sim 190 \mu \mathrm{m}$, with a large fraction of them having diameters of the order of $50 \mu \mathrm{m}$. As they move downstream, the distributions shift toward smaller sizes and at $d=8 \mathrm{~cm}$ they have diameters $<100 \mu \mathrm{m}$, with a major fraction having sizes smaller than $50 \mu \mathrm{m}$. The distributions are well fitted by a lognormal function (continuous, red line). Note that the measured viscosity of this viscoelastic, shear thinning PEO solution is in the range $\sim 0.01 \mathrm{~Pa} \cdot s$. As measured in this work and reported by others, the viscoelastic nature of PEO solutions increases with the molecular weight of the polymer.

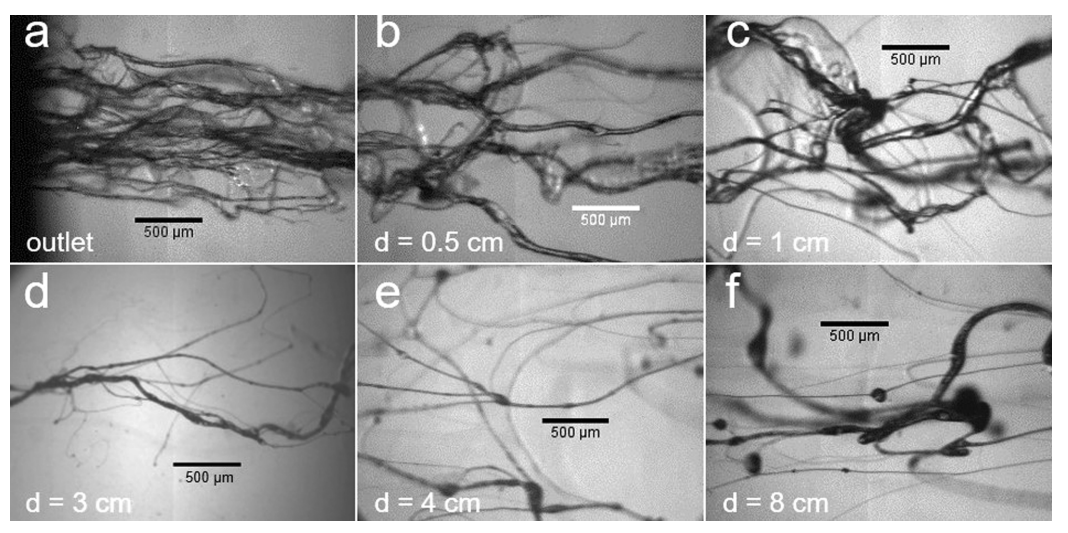

Figure 5. Images of the atomization of PEO 600k $0.8 \mathrm{wt} \%$, with $P_{\mathrm{g}}=4 \mathrm{bar}$ and $P_{1}=3.3 \mathrm{bar}$, as a function of the distance from the atomizer (a) outlet, (b) $d=0.5 \mathrm{~cm},(\mathrm{c}) d=1 \mathrm{~cm},(\mathrm{~d}) d=3 \mathrm{~cm},(\mathrm{e}) d=4 \mathrm{~cm}$, and (f) $d=8 \mathrm{~cm}$. 


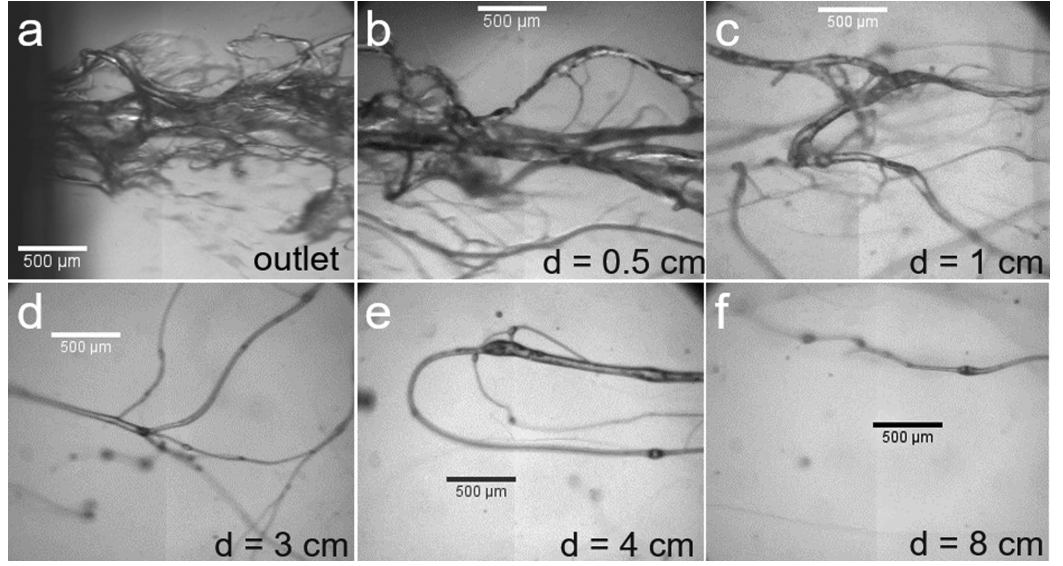

Figure 7. Images of the atomization of aqueous solution of PEO $4 \mathrm{M} 0.2 \mathrm{wt} \%$, with $P_{\mathrm{g}}=4$ bar and $P_{1}=3.3$ bar, as a function of the distance from the atomizer (a) outlet, (b) $d=0.5 \mathrm{~cm},(\mathrm{c}) d=1 \mathrm{~cm},(\mathrm{~d}) d=3 \mathrm{~cm},(\mathrm{e}) d=4 \mathrm{~cm}$, and (f) $d=8 \mathrm{~cm}$.

In general, the viscosity gradient as a function of shear rate is larger for PEO $4 \mathrm{M}$ than for PEO 600k. It is thus expected that aqueous solutions of PEO 4M will exhibit a stronger shear thinning behavior when atomized under the same gas and liquid pressure conditions than the solutions of PEO 600k. In the current work, the solution of PEO $4 \mathrm{M} 0.2$ wt \% had a measured viscosity of $\sim 0.05 \mathrm{~Pa} \cdot \mathrm{s}$, half that of the solution of PEO 600k $0.8 \mathrm{wt} \%$. Figure 7 shows images of a typical atomization of an aqueous solution of PEO 4M 0.2 wt \% recorded at varying distances from the atomizer outlet. Overall, the filament bundle dynamics is similar as in the case of the $600 \mathrm{k}$ solution; that is, the diameter of the liquid ligament decreases as it moves downstream. From Figure 8 it is evident

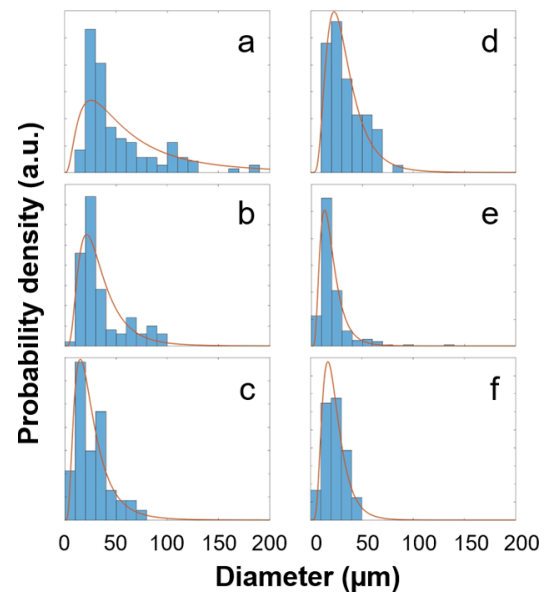

Figure 8. Size distribution of filaments from PEO $4 \mathrm{M} 0.2 \mathrm{wt} \%$ atomized with an FB device at $P_{\mathrm{g}}=4$ bar and $P_{1}=3.3$ bar. $d=$ (a) 0.5 , (b) 1, (c) 2, (d) 3, (e) 4, and (f) $8 \mathrm{~cm}$.

that whereas at $d=0.5 \mathrm{~cm}$ the filaments' diameters are in the range $10-190 \mu \mathrm{m}$, at $d=8 \mathrm{~cm}$ all diameters are below $50 \mu \mathrm{m}$. Note also that for a given $d$, the mean diameter becomes slightly larger with increasing the polymer molecular weight as observed from Figure $8(d \geq 0.5 \mathrm{~cm})$, for the $4 \mathrm{M}$ case, compared to the filaments from 600k PEO (Figure 6). This trend was observed for all atomized solutions. Nevertheless, large filaments observed in the proximity of the atomizer outlet in the case of the $600 \mathrm{k}$ solution (for instance at $1 \mathrm{~cm}, 100-150$ $\mu \mathrm{m})$ are not present in the case of the $4 \mathrm{M}$ PEO, perhaps because of its increasing shear thinning nature. The so-called
Deborah numbers $(\mathrm{De})$, the ratio of a time to relaxation to a "time of observation", of each of the atomized solutions are summarized in Table 1; for this calculation the atomizer's orifice radius $R(=D / 2)$ was used as the characteristic length. That is, $D e=\lambda_{\mathrm{z}} / \sqrt{\rho R^{3} / \sigma}$, where $\rho$ is the density of the solution, $\sigma$ is the surface tension, and $\lambda_{\mathrm{z}}$ is the longest relaxation time in the Rouse-Zimm model. Although such a model is only valid for dilute polymeric solutions, the atomized solutions' $c / c^{*} \approx o(1)$, and thus it may be used as a first approximation. The relaxation time was then estimated with $\lambda_{\mathrm{z}} \cong \frac{1}{\zeta(3 \nu)} \frac{[\eta] M_{\mathrm{v}} \eta_{\mathrm{s}}}{N_{\mathrm{A}} k_{\mathrm{B}} T}$ as applied by other authors, ${ }^{50,51}$ where $N_{\mathrm{A}}$ is Avogadro's number, $k_{\mathrm{B}}$ is the Boltzmann constant $(1.38 \times$ $\left.10^{-23} \mathrm{~m}^{2} \mathrm{~kg} \mathrm{~s}^{-2} \mathrm{~K}^{-1}\right), T$ is the absolute temperature, and $\eta_{\mathrm{s}}$ is the solvent viscosity; in the limit of dominant hydrodynamic interactions $1 / \zeta(3 \nu)$ is 0.422 , and the intrinsic viscosity was estimated with $[\eta]=0.0125 M_{\mathrm{v}}^{0.78}$ in units of $\mathrm{mL} / \mathrm{g}$, for PEO in water. $^{43,52}$ The data show there is approximately three orders of magnitude difference in the relaxation time between PEO 100k and PEO 4M solutions. Furthermore, for PEO 100k (7 wt \%) $\mathrm{De} \ll 1$, thus indicating the lower viscoelastic nature of the filaments and a more Newtonian-like behavior. Conversely, De increases as PEO molecular weight increases, despite a decrease in zero-shear rate viscosity, which is indicative of the remarkably strong viscoelastic behavior of the solutions, particularly the 0.2 wt \% PEO $4 \mathrm{M}$. It is thus reasonable to think that both the existence of filaments at distances far from the emission point and the larger mean filament diameter as PEO molecular weight increases are the result of the viscoelastic rheology of the polymer solutions and the relatively slow relaxation rate.

All atomized solutions (see Table 1) exhibit two types of behaviors, which are depicted in Figure 9. The figure shows the zero-shear rate viscosity as a function of solution composition (polymer mass fraction) for PEO of different molecular weights, in the range $100 \mathrm{k}$ to $4 \mathrm{M}$. For relatively low values of $\eta_{0}$, the atomized solutions formed droplets (data not shown), as in the classical FB mechanism. Such result is in agreement with observations by others during air-blast atomization of dilute polymeric solutions, in which the concentration is well below $c^{*}$. However, the figure also depicts an abrupt change in the slope as the mass fraction is increased above $c_{\mathrm{t}}$. Beyond such value, a linear $\log$-log relationship is observed, as indicated by the straight lines (to guide the eye only) and the 


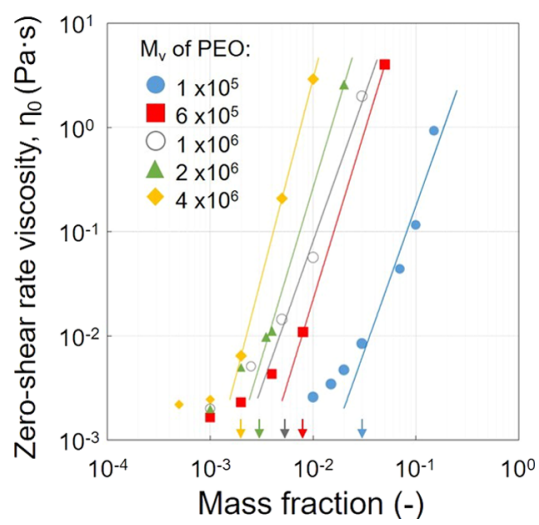

Figure 9. Viscosity at zero-shear rate of PEO solutions as a function of mass concentration. The arrows on the $x$-axis indicate the value of $c^{*}$ for each PEO molecular weight.

production of filaments took place for all $P_{\mathrm{g}}$ used. Such mass fraction coincides with the calculated $c^{*}$ of each solution, indicated by the arrows on the $x$-axis (each color corresponds to a specific PEO $M_{\mathrm{v}}$ ). The filamentary structures were present at $d=8 \mathrm{~cm}$, although in some cases droplets were also observed at such a distance.

We have also performed an analysis of the parameters that govern the atomization process and calculated the dimensionless Ohnesorge number $(\mathrm{Oh})$, which represents the ratio of viscous forces to inertio-capillary forces, and given by $O h=\eta_{0} / \sqrt{\rho \sigma d_{\mathrm{j}}}$. Figure $\mathrm{S} 1$ in the Supporting Information file shows a dimensionless scaling parameter that includes the $\mathrm{Oh}$ as a function of gas pressure for PEO solutions at the threshold concentration that resulted in filaments (Table 1). The parameter is $\operatorname{Oh}\left(M_{\mathrm{v}} / 10^{5} M_{\mathrm{o}}\right)^{0.33} c_{\mathrm{m}}{ }^{-0.35}$, where $M_{\mathrm{o}}$ is the molecular weight of the monomer and $c_{\mathrm{m}}$ is the mass fraction of the polymer in solution at $c_{\mathrm{t}}$. Note that the parameter takes into account the effect of polymer molecular weight and concentration. At a relatively low pressure, the scaling parameter is slightly below unity for PEO of all molecular weights. However, at $P_{\mathrm{g}}=4 \mathrm{bar}$, it appears as though the scaling parameter reaches, first, a maximum and then a plateau around unity. The graph shows that the data points tend to collapse around the scaling parameter. Also note that in the case of PEO 100k the Oh would be higher than 1, thus indicating that viscous forces dominate the process. Conversely, in the case of PEO of high molecular weight $(\geq 600 \mathrm{k})$ the $O h$ would be smaller than $1(>0.3)$ for all $P_{g}$, perhaps because of their marked shear thinning nature compared with PEO 100k.

\section{CONCLUSIONS}

We have applied the FB method to massively generate liquid filaments. The test liquids consisted of solutions of $\mathrm{PEO}$ of varying molecular weights, in the range $100 \mathrm{k}$ to $4 \mathrm{M}$, as well as varying concentrations. Not all PEO solutions resulted in generation of filaments, but only those above a threshold concentration value, which sets the onset for liquid ligament formation for each molecular weight; such concentration appears to be the well-known overlap concentration $c^{*}$ of polymer coils. The threshold concentration increased with decreasing PEO molecular weight. For instance, for PEO of the lowest molecular weight, $100 \mathrm{k}$, the threshold concentration was $7 \mathrm{wt} \%$, whereas for $4 \mathrm{M}$ such value decreased to $0.2 \mathrm{wt} \%$.
When solutions of concentrations lower than $c^{*}$ were atomized, the typical functioning mode of the FB was achieved, that is, sprays were produced. Except for solutions of PEO 100k, all solutions exhibited a marked shear thinning behavior. The droplet production and filament production regimes were characterized using a Bueche parameter, that is, the product of the mass fraction of PEO in the solution times the ratio of viscosity-average molecular weight of $\mathrm{PEO}$ to its entanglement molecular weight. In our experiments, the critical Bueche parameter was $o(1)$. A 3.2-dependency of the zeroshear rate viscosity on the Bueche parameter was found for filament production, whereas a nearly linear dependency was found for droplet production. As a general trend, the mean filament diameter decreased as a function of the distance from the atomizer outlet. Furthermore, for a given gas pressure the mean filament diameter increased with the rise of the PEO molecular weight. This work sets a general and energetically efficient approach for the massive production of liquid filaments, at a rate of the order of tens of liters per hour, for an immense variety of scientific and industrial applications such as biomedical scaffolds, filters, fabrics, mats, paper, packaging stuff, artificial hydroponic or aeroponic soils or substrates, and so forth. These fibrous materials may thus be synthesized with our FB-enabled approach by adding a postprocessing procedure, for instance, an on-line drying step following filament generation. Conversely, an off-line step may be used as well. In such a case, the filaments would be collected onto a substrate and then calcined in an oven.

\section{ASSOCIATED CONTENT}

\section{Supporting Information}

The Supporting Information is available free of charge on the ACS Publications website at DOI: 10.1021/acsomega.8b02542.

$$
\begin{aligned}
& \text { Graph of dimensionless parameter, } \\
& \operatorname{Oh}\left(\frac{M_{\mathrm{v}}}{10^{5} M_{\mathrm{o}}}\right)^{0.33} c_{\mathrm{m}}^{-0.35} \text {, as a function of gas pressure } \\
& (\mathrm{PDF})
\end{aligned}
$$

\section{AUTHOR INFORMATION}

\section{Corresponding Author}

*E-mail: lmodesto@us.es. Phone: +34 (954) 48-7224 (L.B.M.L.).

\section{ORCID}

Luis B. Modesto-López: 0000-0002-9493-8846 Alfonso M. Gañán-Calvo: 0000-0002-7552-6184

\section{Notes}

The authors declare no competing financial interest.

\section{ACKNOWLEDGMENTS}

L.B.M.-L. and A.M.G.-C. acknowledge financial support from the Ministerio de Economía y Competitividad (Spain), Plan Estatal 2013-2016 Retos, project DPI2016-78887-C3-1-R.

\section{REFERENCES}

(1) Cooper, J. A.; Lu, H. H.; Ko, F. K.; Freeman, J. W.; Laurencin, C. T. Fiber-Based Tissue-Engineered Scaffold for Ligament Replacement: Design Considerations and in Vitro Evaluation. Biomaterials 2005, 26, 1523-1532. 
(2) Lutolf, M. P.; Hubbell, J. A. Synthetic Biomaterials as Instructive Extracellular Microenvironments for Morphogenesis in Tissue Engineering. Nat. Biotechnol. 2005, 23, 47.

(3) Chen, J.; Huang, Y.; Zhang, N.; Zou, H.; Liu, R.; Tao, C.; Fan, X.; Wang, Z. L. Micro-Cable Structured Textile for Simultaneously Harvesting Solar and Mechanical Energy. Nat. Energy 2016, 1, 1-8.

(4) Nogi, M.; Iwamoto, S.; Nakagaito, A. N.; Yano, H. Optically Transparent Nanofiber Paper. Adv. Mater. 2009, 21, 1595-1598.

(5) Modesto-López, L. B.; Chimentão, R. J.; Álvarez, M. G.; RosellLlompart, J.; Medina, F.; Llorca, J. Direct Growth of Hydrotalcite Nanolayers on Carbon Fibers by Electrospinning. Appl. Clay Sci. 2014, 101, 461-467.

(6) Kapantaidakis, G. C.; Koops, G. H. High flux polyethersulfonepolyimide blend hollow fiber membranes for gas separation. J. Memb. Sci. 2002, 204, 153-171.

(7) Alexiadis, A.; Alberini, F.; Meyer, M. E. Geopolymers from Lunar and Martian Soil Simulants. Adv. Sp. Res. 2017, 59, 490-495.

(8) Chen, X.; Zhu, G.; Zhou, M.; Wang, J.; Chen, Q. Effect of Organic Polymers on the Properties of Slag-Based Geopolymers. Constr. Build. Mater. 2018, 167, 216-224.

(9) Khadka, D. B.; Haynie, D. T. Insoluble Synthetic Polypeptide Mats from Aqueous Solution by Electrospinning. ACS Appl. Mater. Interfaces 2010, 2, 2728-2732.

(10) Bessaire, B.; Mathieu, M.; Salles, V.; Yeghoyan, T.; Celle, C.; Simonato, J.-P.; Brioude, A. Synthesis of Continuous Conductive PEDOT:PSS Nanofibers by Electrospinning: A Conformal Coating for Optoelectronics. ACS Appl. Mater. Interfaces 2016, 9, 950-957.

(11) Gañán-Calvo, A. M. Cone-Jet Analytical Extension of Taylor's Electrostatic Solution and the Asymptotic Universal Scaling Laws in Electrospraying. Phys. Rev. Lett. 1997, 79, 217-220.

(12) Dror, Y.; Salalha, W.; Khalfin, R. L.; Cohen, Y.; Yarin, A. L.; Zussman, E. Carbon Nanotubes Embedded in Oriented Polymer Nanofibers by Electrospinning. Langmuir 2003, 19, 7012-7020.

(13) Li, D.; Xia, Y. Electrospinning of Nanofibers: Reinventing the Wheel? Adv. Mater. 2004, 16, 1151-1170.

(14) Di, J.; Zhao, Y.; Yu, J. Fabrication of Molecular Sieve Fibers by Electrospinning. J. Mater. Chem. 2011, 21, 8511.

(15) Mohamed, A. S.; Lopez-Herrera, J. M.; Herrada, M. A.; Modesto-Lopez, L. B.; Gañán-Calvo, A. M. Effect of a Surrounding Liquid Environment on the Electrical Disruption of Pendant Droplets. Langmuir 2016, 32, 6815-6824.

(16) Ding, H.; Zhu, C.; Tian, L.; Liu, C.; Fu, G.; Shang, L.; Gu, Z. Structural Color Patterns by Electrohydrodynamic Jet Printed Photonic Crystals. ACS Appl. Mater. Interfaces 2017, 9, 11933-11941.

(17) Modesto-Lopez, L. B.; Thimsen, E. J.; Collins, A. M.; Blankenship, R. E.; Biswas, P. Electrospray-Assisted Characterization and Deposition of Chlorosomes to Fabricate a Biomimetic LightHarvesting Device. Energy Environ. Sci. 2010, 3, 216-222.

(18) Gañán-Calvo, A. M. Enhanced Liquid Atomization: From Flow-Focusing to Flow-Blurring. Appl. Phys. Lett. 2005, 86, 214101.

(19) Rosell-Llompart, J.; Gañán-Calvo, A. M. Turbulence in Pneumatic Flow Focusing and Flow Blurring Regimes. Phys. Rev. E - Stat. Nonlinear, Soft Matter Phys. 2008, 77, 1-10.

(20) Wei, S.; Qu, G.; Luo, G.; Huang, Y.; Zhang, H.; Zhou, X.; Wang, L.; Liu, Z.; Kong, T. Scalable and Automated Fabrication of Conductive Tough-Hydrogel Microfibers with Ultrastretchability, 3D Printability, and Stress Sensitivity. ACS Appl. Mater. Interfaces 2018, 10, 11204-11212.

(21) Modesto-López, L. B.; Gañán-Calvo, A. M. Visualization and size-measurement of droplets generated by Flow Blurring in a highpressure environment. Aerosol Sci. Technol. 2017, 52, 198-208.

(22) Simmons, B. M.; Agrawal, A. K. Flow Blurring Atomization for Low-Emission Combustion of Liquid Biofuels. Combust. Sci. Technol. 2012, 184, 660-675.

(23) Jiang, L.; Agrawal, A. K. Investigation of Glycerol Atomization in the Near-Field of a Flow-Blurring Injector using Time-Resolved PIV and High-Speed Visualization. Turbul. Combust. 2014, 94, 323338.
(24) Niguse, Y.; Agrawal, A. Low-Emission, Liquid Fuel Combustion System for Conventional and Alternative Fuels Developed by the Scaling Analysis. J. Eng. Gas Turbines Power 2015, 138, 041502.

(25) Tuttle, S. G.; Farley, J. P.; Fleming, J. W. Efficient Atomization and Combustion of Emulsified Crude Oil; Naval Research Laboratory: Washington, DC, USA, 2014.

(26) Petrie, C. J. S. Extensional Viscosity: A Critical Discussion. J. Non-Newtonian Fluid Mech. 2006, 137, 15-23.

(27) McKinley, G. H. Visco-Elasto-Capillary Thinning and Break-Up of Complex Fluids. In Rheology Reviews; Binding, D. M.; Walters, K., Eds.; British Society of Rheology: Aberystwyth, U.K., 2005; pp 1-49.

(28) Barnes, H. A.; Hutton, J. F.; Walter, K. An Introduction to Rheology, 1st ed.; Elsevier Science Publishers B.V.: Amsterdam, 1989.

(29) Keshavarz, B.; Houze, E. C.; Moore, J. R.; Koerner, M. R.; McKinley, G. H. Ligament Mediated Fragmentation of Viscoelastic Liquids. Phys. Rev. Lett. 2016, 117, 154502.

(30) Lysak, I. A.; Malinovskaya, T. D.; Lysak, G. V.; Potekaev, A. I.; Kulagina, V. V.; Tazin, D. I. Formation of Fiber Materials by Pneumatic Spraying of Polymers in Viscous-Flow States. Russ. Phys. J. 2017, 59, 1581-1588.

(31) Otaigbe, J. U.; McAvoy, J. M. Gas Atomization of Polymers. I. Feasibility Studies and Process Development. Adv. Polym. Technol. 1998, 17, 145-160.

(32) Li, L. K. B. An Experimental Study on Air-Blast Atomization of Viscoelastic Liquids; The University of British Columbia, 2006.

(33) Daristotle, J. L.; Behrens, A. M.; Sandler, A. D.; Kofinas, P. A Review of the Fundamental Principles and Applications of Solution Blow Spinning. ACS Appl. Mater. Interfaces 2016, 8, 34951-34963.

(34) Abràmoff, M. D.; Magalhães, P. J.; Ram, S. J. Image Processing with ImageJ. Biophotonics Int. 2004, 11, 36-42.

(35) Ebagninin, K. W.; Benchabane, A.; Bekkour, K. Rheological Characterization of Poly(Ethylene Oxide) Solutions of Different Molecular Weights. J. Colloid Interface Sci. 2009, 336, 360-367.

(36) Grigorescu, G.; Kulicke, W.-M. Viscoelasticity, Atomistic Models, Statistical Chemistry; Springer, 2000; Vol. 152, pp 1-40.

(37) Bouldin, M.; Kulicke, W.-M.; Kehler, H. Prediction of the NonNewtonian Viscosity and Shear Stability of Polymer Solutions. Colloid Polym. Sci. 1988, 266, 793-805.

(38) Larson, R. G. The Rheology of Dilute Solutions of Flexible Polymers: Progress and Problems. J. Rheol. 2005, 49, 1-70.

(39) Colby, R. H.; Boris, D. C.; Krause, W. E.; Dou, S. Shear Thinning of Unentangled Flexible Polymer Liquids. Rheol. Acta 2006, 46, 569-575.

(40) Kulicke, W.-M.; Clasen, C. Viscosimetry of Polymers and Polyelectrolytes; Springer-Verlag: Berlin, 2005.

(41) Clasen, C.; Plog, J. P.; Kulicke, W.-M.; Owens, M.; Macosko, C.; Scriven, L. E.; Verani, M.; McKinley, G. H. How Dilute Are Dilute Solutions in Extensional Flows? J. Rheol. 2006, 50, 849-881.

(42) Devanand, K.; Selser, J. C. Asymptotic Behavior and LongRange Interactions in Aqueous Solutions of Poly(Ethylene Oxide). Macromolecules 1991, 24, 5943-5947.

(43) Dinic, J.; Biagioli, M.; Sharma, V. Pinch-off Dynamics and Extensional Relaxation Times of Intrinsically Semi-Dilute Polymer Solutions Characterized by Dripping-onto-Substrate Rheometry. J. Polym. Sci. Part B Polym. Phys. 2017, 55, 1692-1704.

(44) Palangetic, L.; Reddy, N. K.; Srinivasan, S.; Cohen, R. E.; McKinley, G. H.; Clasen, C. Dispersity and Spinnability: Why Highly Polydisperse Polymer Solutions Are Desirable for Electrospinning. Polymer 2014, 55, 4920-4931.

(45) Heo, Y.; Larson, R. G. Universal Scaling of Linear and Nonlinear Rheological Properties of Semidilute and Concentrated Polymer Solutions. Macromolecules 2008, 41, 8903-8915.

(46) Dontula, P.; Macosko, C. W.; Scriven, L. E. Model Elastic Liquids with Water-Soluble Polymers. AIChE J. 1998, 44, 1247-1255.

(47) Shenoy, S. L.; Bates, W. D.; Frisch, H. L.; Wnek, G. E. Role of Chain Entanglements on Fiber Formation during Electrospinning of Polymer Solutions: Good Solvent, Non-Specific Polymer-Polymer Interaction Limit. Polymer 2005, 46, 3372-3384. 
(48) Heo, Y.; Larson, R. G. The Scaling of Zero-Shear Viscosities of Semidilute Polymer Solutions with Concentration. J. Rheol. 2005, 49, $1117-1128$

(49) Raspaud, E.; Lairez, D.; Adam, M. On the Number of Blobs per Entanglement in Semidilute and Good Solvent Solution: Melt Influence. Macromolecules 1995, 28, 927-933.

(50) Tirtaatmadja, V.; McKinley, H. G.; Cooper-White, J. J. Drop Formation and Breakup of Low Viscosity Elastic Fluids: Effects of Molecular Weight and Concentration. Phys. Fluids 2006, 18, 043101.

(51) Greiciunas, E.; Wong, J.; Gorbatenko, I.; Hall, J.; Wilson, M. C. T.; Kapur, N.; Harlen, O. G.; Vadillo, D.; Threlfall-Holmes, P. Design and Operation of a Rayleigh Ohnesorge Jetting Extensional Rheometer (ROJER) to Study Extensional Properties of Low Viscosity Polymer Solutions. J. Rheol. 2017, 61, 467.

(52) Mark, J. E. Polymer Data Handbook; Oxford University Press, Inc.: New York, 1999. 